\title{
Electrode stress device for electrochemical power
}

\author{
Wenzhuo $\mathrm{Wu}$ \\ NPG Asia Materials (2016) 8, e267; doi:10.1038/am.2016.49; published online 13 May 2016
}

Mechanical vibrational energy is ubiquitous and abundant throughout the ambient environment for powering the sustainable operations of integrated electronics and sensors in biomedical devices, wearable systems and robotics. Writing in Nature Communications, ${ }^{1} \mathrm{Ju} \mathrm{Li}$ et al. at MIT now report on the development of an electrochemically driven energy harvester for scavenging energy from mechanical motion.

Many approaches have been adopted to harvest mechanical energy including electromagnetic induction, electrostatic generation, piezoelectric and triboelectric effects. ${ }^{2}$ In all these technologies, the conversion from mechanical energy to electrical power involves a physical process. As an example, in a piezoelectric harvester, electrical charges are induced in the strained piezoelectric materials due to the lack of inversion symmetry in the material's crystal structure. In a triboelectric energy harvester, the mechanically-induced triboelectrification between two dissimilar surfaces gives rise to a voltage.

In contrast, Ju Li et al. demonstrated a novel approach to convert mechanical energy through an electrochemical route using a simple design and device structure that consists of two identical Li-alloyed Si electrodes and an electrolyte-soaked separator membrane. ${ }^{1}$ Conventionally, the coupling between the mechanical stress and lithiation process is regarded as an adverse effect that occurs in the high-capacity anodes of lithium ion batteries. ${ }^{3,4}$ In their work, ${ }^{1} \mathrm{Li}$ et al. utilized bending-induced asymmetric stresses in the two electrodes to generate a chemical potential difference that can drive the flux of lithium ions from the compressed to the stretched electrode (Figure 1). The corresponding electron flow in the exter|nal circuit gives rise to a voltage. This design and experimental approach is promising for practical use in harvesting mechanical energy from sources such as human activities and ambient vibrations.

There are very few reports on any similar concepts or designs. Although the mechanicallydriven migration of lithium ions based on

School of Industrial Engineering, Purdue University, West Lafayette, IN, USA

E-mail: wenzhuowu@purdue.edu

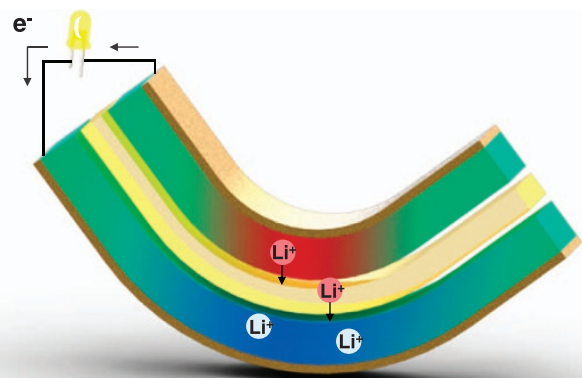

Figure 1 Schematic illustration of the underlying principle of the electrochemically driven energy harvester (Courtesy of Ju Li, MIT).

the piezoelectric effect has been previously demonstrated for directly converting mechanical energy into chemical energy, ${ }^{5}$ Li's work utilizes a simpler device design based on stress-voltage coupling in an electrochemical process. More significantly, the output current from the demonstrated device is large with a long pulse duration, which is beneficial for practical applications. Small output currents with short pulse durations are usually the major limitation seen for other types of mechanical energy harvesters. Moreover, such an electrochemically driven mechanical energy harvester is efficient at scavenging very low-frequency mechanical signals from the ambient environment, for example, the human body, which are largely ignored and wasted in previous studies.

This work has potential in many applications, such as flexible electronics, self-powered sensors, wearable devices, robotics, artificial skin and so on. Future work may involve acquiring a better understanding of the fundamental issues associated with the observed large current and small voltage, and improving the voltage output. It may also be interesting to see if this type of device is feasible for use as a battery that is capable of directly storing any mechanical energy harvested from the ambient environment.

\section{CONFLICT OF INTEREST}

The author declares no conflict of interest.
1 Kim, S., Choi, S. J., Zhao, K., Yang, H., Gobbi, G., Zhang, S. \& Li, J. Electrochemically driven mechanical energy harvesting. Nat. Commun. 7, 10146 (2016).

2 Fan, F. R., Tang, W. \& Wang, Z. L. Flexible nanogenerators for energy harvesting and self-powered electronics. Adv. Mater. (e-pub ahead of print 7 January 2016; doi:10.1002/adma.201504299).

3 Sethuraman, V. A., Srinivasan, V., Bower, A. F. \& Guduru, P. R. In situ measurements of stresspotential coupling in lithiated silicon. J Electrochem Soc 157, A1253-A1261 (2010).

4 Zhao, K., Wang, W. L., Gregoire, J., Pharr, M., Suo, Z., Vlassak, J. J. \& Kaxiras, E. Lithium-assisted plastic deformation of silicon electrodes in lithium-ion batteries: a first-principles theoretical study. Nano. Lett. 11, 2962-2967 (2011).

5 Xue, X., Wang, S., Guo, W., Zhang, Y. \& Wang, Z. L. Hybridizing energy conversion and storage in a mechanical-to-electrochemical process for self-charging power cell. Nano. Lett. 12, 5048-5054 (2012).

(c) (i) This work is licensed under a Creative Commons Attribution 4.0 International License. The images or other third party material in this article are included in the article's Creative Commons license, unless indicated otherwise in the credit line; if the material is not included under the Creative Commons license, users will need to obtain permission from the license holder to reproduce the material. To view a copy of this license, visit http:// creativecommons.org/licenses/by/4.0/ 